\title{
TEMA 2017: Efectividad y seguridad de la quimioterapia de inducción en el tratamiento del Cáncer de colon en el Hos- pital San Juan de Dios durante los años 2010 y 2011
}
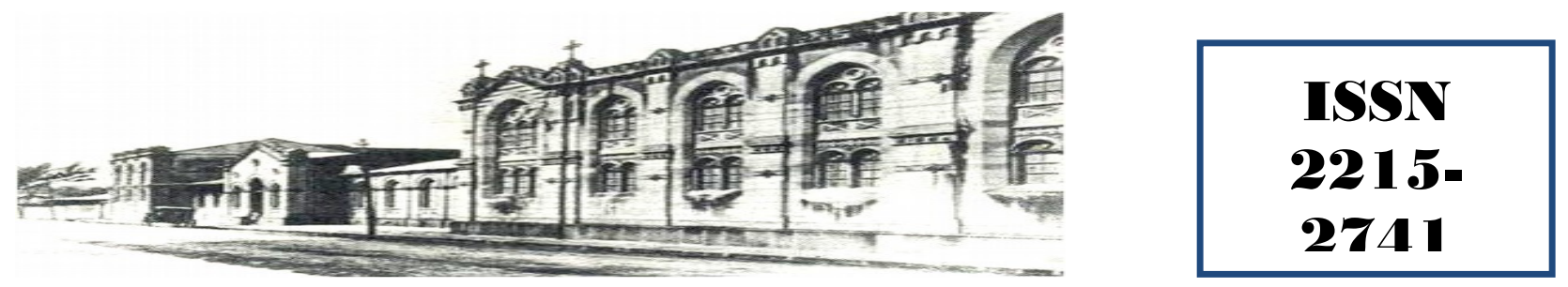

Haspital San quan de Dias. San José. Casta Rica. Fundada en 1845

Recibido:

$10 / 07 / 2017$

Aceptado:

Allan Ramos Esquivel ${ }^{1}$ Cristina Fernandez Barrantes ${ }^{2}$

Denisse Vargas Matarrita ${ }^{3}$

Eugenia Cordero García ${ }^{4}$

\footnotetext{
${ }^{1}$ MSc. Hospital San Juan de Dios. Servicio de Oncología. San Jose, Costa Rica. allanramoscr@gmail.com

${ }^{2}$ MSc. Hospital San Juan de Dios. Servicio de Farmacia. San José, Costa Rica. cristifdez@gmail.com

${ }^{2}$ HSJD- UCR. Estudiante de Internado en Farmacia. San Pedro, Costa Rica

${ }^{4}$ Msc. Universidad de Costa Rica. Facultad de Farmacia. San Pedro, Costa Rica. eugenia.corderogarcia@ucr.ac.cr
}

\section{RESUMEN}

La neoplasia colorrectal es un proceso maligno que implica varias alteraciones genéticas y fenotípicas que favorecen la proliferación de células malignas que terminan generando una lesión rígida que puede ser detectada por técnicas como la proctoscopia. El presente estudio analiza los posibles beneficios de la quimioterapia de inducción en pacientes diagnosticados con este tumor. Se realizó un análisis retrospectivo de 20 pacientes tratados del año 2010 al 2011, en donde la mediana de seguimiento fue de 69.5 meses. La quimioterapia de inducción se le aplicó al 30\% de la población del estudio. Aunque no hay diferencias estadísticamente significativas en la sobrevida entre los dos grupos, se demostró un "downstaging" positivo en un $30 \%$ de la población estudiada y la ausencia de la recurrencias locales y sistémicas en el $80 \%$ de los pacientes.

\section{PALABRAS CLAVE}

Quimioterapia de inducción; quimioradioterapia; neoplasia de recto 
Colorectal neoplasia is a malignant process involving several genetic and phenotypic alterations favoring the proliferation of malignant cells that end up generating a rigid lesion can be detected by techniques such as proctoscopy. This study analyzes the potential benefits of induction chemotherapy in patients diagnosed with this tumor. A retrospective analysis of 20 patients treated from 2010 to 2011, where the median follow-up was 69.5 months was performed. Induction chemotherapy was applied to $30 \%$ of the study population. Although no statistically significant differences in survival for patients receiving induction chemotherapy and that a "downstaging" positive no, it was shown by $30 \%$ of the study population and the absence of local and systemic recurrences in $80 \%$ from the patients.

\section{KEY WORDS}

Induction chemotherapy; chemoradiotherapy; rectal neoplasms.

\section{INTRODUCCIÓN}

El desarrollo de una neoplasia colorrectal es un proceso que implica varias alteraciones genéticas y fenotípicas de la estructura normal de epitelio intestinal así como de su función, esto lleva al crecimiento celular sin regulación y acelerado, así como a la proliferación y el desarrollo de tumores. ${ }^{(1,2)}$ Características de la tumorigénesis colorrectal incluyen la inestabilidad genómica, la activación de las vías de oncogenes, la inactivación mutacional de genes supresores de tumores, la inestabilidad microsatelital y la activación de las vías del factor de crecimiento. ${ }^{(3)}$ Los elementos clave de este proceso incluyen la hiperproliferación de las células epiteliales para formar una pequeña neoplasia benigna o adenoma sumando a la adquisición de varias mutaciones genéticas ${ }^{(1-}$ 4). Debido a esta proliferación crónica es que las células cancerígenas adquieren la capacidad de producir señales de crecimiento ya sea al producir ellas mismas ligandos de crecimiento que generan la producción de receptores afines que produce estimulación autocrina proliferativa o al enviar señales que estiman a las células normales que terminan por serie de procesos supliendo de manera recíproca a las células cancerígenas con factores de crecimiento ${ }^{(3)}$.
La definición anatómica del recto es muy variable, ya que se utilizan tanto la definición endoscópica como los criterios intraoperatorias para definirlo. ${ }^{(4)}$ La proctoscopia rígida es un método altamente reproducible para determinar el nivel del recto y es menos dependiente del operador o de la técnica. Con esta técnica el margen anal es el punto preferido de referencia, puesto que el borde del tumor y el margen anal puede ser visualizado de forma simultánea durante proctoscopia rígida. ${ }^{(1,2,4,5)}$ De esta manera el cáncer de recto se define como una lesión localizada a los $12 \mathrm{~cm}$ del canal anal utilizando la técnica de proctoscopia rígida. ${ }^{(1,2,4,6)}$

La clasificación del estadio de la enfermedad en cáncer de recto es de suma importancia ya que es utilizado directamente para determinar el tratamiento que se utilizará en cada paciente. ${ }^{(4,6-7)}$

Una vez se ha diagnosticado el cáncer colorectal, se deben tomar en cuenta ciertas consideraciones para el tratamiento que incluyen la probabilidad de mantener o restaurar la función normal del intestino o la continencia anal y preservar las funciones genitourinarias. (1,4) La guía de la NCCN ha indicado que pacientes con estadio T3N0, TxN1-2 o incluso T4 deben ser tratados con quimioradioterapia preoperatoria y cirugía. Incluso esta guía indica que los pacientes que se encuentran en estadio T3N0M0, que presentan un tumor con márgenes claros y pronóstico favorable, tienen bajas posibilidades de recurrencia. Estos paciente pueden ser tratados adecuadamente por medio de la cirugía y la quimioterapia adyuvante. ${ }^{(4,8-12)}$

La terapia que utiliza la combinación de quimioterapia con compuestos a base de fluoropirimidinas junto con radioterapia, cirugía y quimioterapia neoadyuvante es recomendada en la mayoría de los pacientes en estadio II o III. En estos pacientes se recomiendan dos opciones: quimioradioterapia preoperatoria seguida de quimioterapia postoperatoria, o quimioterapia seguida de quimoradioterapia y luego resección. ${ }^{(4,7-9,11,13-14)}$ El tratamiento quimioterapéutico indicado durante la radioterapia es monoterapia con una fluoro-

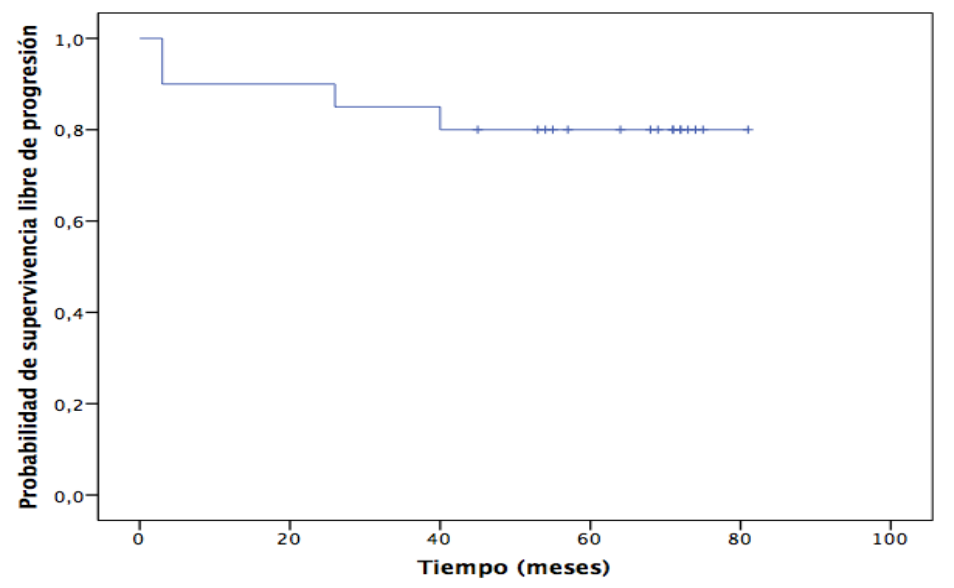


pirimidina (capecitabina oral o 5-flurouracilo) siempre y cuando no exista contraindicación para su administración, además de la adición de leucovorina. ${ }^{(4,7)}$ El tratamiento quimioterapéutico adyuvante postoperatorio sigue las mismas recomendaciones que para el colon estadios II y III, $(4,7,14)$ de hecho este tratamiento se utiliza ampliamente debido a la reducción significativa en la recidiva local y el menor número de efectos tóxicos así como la preservación del esfínter, lo cual ha mostrado ser mayor en estos pacientes que en los que reciben quimiorradiación postoperatoria. (1) En los pacientes intervenidos estadios II y III que no han recibido tratamiento preoperatorio se recomienda tratamiento adyuvante complementario con capecitabina o 5-flurouracilo durante la radioterapia adyuvante, completando posteriormente con quimioterapia con los mismos esquemas que para el colon hasta completar los 6 meses de tratamiento. ${ }^{(4,13)}$

Algunos posibles beneficios de utilizar la quimioterapia de inducción son la erradicación temprana o prevención de micrometastasis, las altas tasas de respuesta patológica completa, la minimización del tiempo en el que los pacientes requieren una ileostomía, la facilitación de la resección y la mejora de la tolerancia para completar los ciclos de quimioterapia. ${ }^{(4,11)}$

En cuanto a la sobrevida el cáncer de recto, tienen una mayor tasa de fracaso local cuando se utiliza la cirugía sola, sobre todo si el tumor está más avanzado. La quimioradioterapia neoadyuvante da como resultado un perfil de toxicidad más favorable a largo plazo y un mejor control local, también puede mejorar la capacidad de realizar la preservación del esfínter en algunos pacientes con cánceres rectales de bajo nivel en el canal rectal. ${ }^{(6,10)}$

Debido a que el uso de quimioterapia de inducción es una práctica de la que se tiene poca evidencia, se planteó para este estudio como objetivo general evaluar la efectividad y seguridad de la quimioterapia de inducción en el tratamiento del cáncer de colon en el Hospital San Juan de Dios (HSJD) durante los años 2010 y 2011. Esto permite caracterizar a la población en estudio, determinar la efectividad de la quimioterapia de inducción en la población en términos de sobrevida global y determinar la seguridad del uso de la quimioterapia de inducción mediante la identificación de reacciones adversas documentadas en el expediente clínico de los pacientes en estudio.

\section{DISCUSIÓN}

Respecto a la edad y sexo de los pacientes no hay diferenciación en cuanto a la incidencia del cáncer colorrectal, y de acuerdo a los datos de la Organización Mundial de la Salud (OMS) se determina que este es el cuarto cáncer de mayor diagnóstico y el segundo en mortalidad en los Estados Unidos. Se estimó que para el año 2015 se diagnosticarían 40.000 nuevos casos de cáncer de recto, un $58 \%$ correspondería a hombres y un $42 \%$ a mujeres, y que aproximadamente 50.310 personas morirían debido al cáncer de recto $\mathrm{y}$ combinaciones del mismo para este mismo período. ${ }^{(4,15)}$

Así mismo, el proyecto GLOBOCAN no diferencia el cáncer colorrectal e indica que en Costa Rica se estimaba que para el 2012 se iban a presentar 819 nuevos casos de cáncer colorectal donde $49 \%$ serían mujeres y $51 \%$ hombres, esta misma predicción se repitió en el 2015, donde del estimado de 924 casos el porcentaje para hombres y mujeres iba a mantenerse, se esperaba que 2012 y 2015 se presentaran más casos de cáncer colorectal en hombres mayores de $65^{\cdot(15)}$

En el Registro Nacional de Tumores se indica que en el 2012 el cáncer de colon fue el cuarto cáncer más común en hombres y el sexto en mujeres, y teniendo un aumento en la tasa de mortalidad desde el año 2000 al 2013 incluso llegando a ser la tercera causa de mortalidad por tumores malignos en mujeres. ${ }^{(16)}$ Sin embargo la población del estudio mostró ser diferente a lo esperado ya que la edad promedio fue menor a 65 años, aunque si se presentaron más casos de hombres que de mujeres.

En relación al valor del ECOG, los estudios han demostrado que si este se encuentra alterado se asociará a un mayor riesgo de toxicidad debido a la quimioterapia. Incluso, en un estudio donde se analizó la respuesta en pacientes mayores de 70 años se logró identificar que un estado funcional de ECOG $\geq 2$ es un predictor específico de la toxicidad a la quimioterapia. ${ }^{(4,17-19)}$

Por la tanto, se podría indicar que los pacientes de este estudio no tenían una alteración en el estado funcional que los hiciera propensos a presentar toxicidad por la quimioterapia en el momento en que iniciaron el tratamiento. 
En relación al tratamiento de los pacientes con cáncer de recto, estudios en fase II como el de Férnadez-Martos et al., que utiliza como referencia los estudios de Rödel et al. y Sebag-Montefiore et al. en pacientes con adenocarcinoma avanzado, donde se ha utilizado capecitabina más oxalipaltino se han obtenido tasas de respuesta patológica completa de $10 \%$ a $19 \%$. ${ }^{(20)}$

Sin embargo, el 5-FU sigue siendo la base de la quimioterapia en pacientes con cáncer colonorectal, ${ }^{(21)}$ por lo tanto se respalda el uso de este tratamiento en $10 \%$ de la población. Así mismo, dos grandes ensayos de fase III referenciados en el estudio de Férnadez-Martos et al., donde se comparó la administración de 5-FU y FOLFOX junto con radiación preoperatoria en pacientes con cáncer rectal localmente avanzado, confirman una respuesta favorable al utilizar cualquier fluropirimidina combinada con radioterapia, ${ }^{(20)}$ por lo que también se apoya el uso de FOLFOX en este estudio.

Aunque cabe destacar que no hay un consenso en referencia al uso de quimioterapia de inducción, las mismas guías indican la existencia de estudios que se contradicen entre sí. Algunos de estos estudios señalan que la quimioterapia de inducción puede causar más toxicidad al paciente, mientras que otros indican que la quimioterapia de inducción es menos tóxica y mejor tolerada. ${ }^{(4)}$ En el caso de este estudio, los pacientes que utilizaron quimioterapia de induccion no presentaron ninguna toxicidad.

El presente estudio mostró que un $60 \%$ de la población que recibió la quimioterapia de inducción fue sometida a una cirugía RAB. Estos mismos resultados fueron observados por Bhatti et al., donde se logró determinar que la preservación del esfínter es posible con mucha más frecuencia en grupos de pacientes que han recibido quimioterapia de inducción. ${ }^{(22)} \mathrm{El}$ 93\% de los pacientes del estudio de Bhatti et al. recibieron quimioterapia de inducción y $100 \%$ recibieron una escisión total del mesorrecto, mientras que de los 61 pacientes que no la recibieron sólo 93,4\% recibieron una escisión total del mesorrecto. (22)

Incluso, el estudio del Hospital Royal Marsden en datos preliminares ha demostrado que, los pacientes de riesgo bajo con cáncer de recto tratados con quimioterapia de inducción a base de capecitabina y oxaliplatino antes de quimioradioterapia concomitante han presentado regresión sus- tancial del tumor, respuesta sintomática rápida, sin progresión de la enfermedad durante el tratamiento preoperatorio, (23) ambos estudios concuerdan con los resultados del presente estudio sin embargo, la población obtenida en el Hospital San Juan de Dios no fue la suficiente como para realizar una comparación estadísticamente significativa entre el grupo que recibió quimioterapia de inducción y el grupo que no la recibió como parte de su esquema de tratamiento.

Se han mostrado estudios donde la administración de combinaciones de FOLFOX y XELODA seguidos de quimiorradiación preoperatoria resulta en la regresión del tumor de manera sustancial, una rápida respuesta sintomática y una no progresión de la enfermedad durante el tratamiento preoperatorio. Así mismo, utilizar quimioterapia de inducción a base de capecitabina antes de la quimioradioterapia ha demostrado ser un enfoque viable, con tasas de respuesta patológica completa hasta $24 \% .^{(24,25)}$ En cuanto a la quimioradiación, también se ha visto resultados favorables en el downstaging, el aumento de la cirugía curativa y la conservación del esfínter, ya que la combinación de radioterapia preoperatoria y cirugía, en comparación con la cirugía sola, también mejora significativamente el control local y la supervivencia global. La quimiorradioterapia preoperatoria posee ventajas potenciales por la aplicación de los dos agentes antes de la operación. ${ }^{(25)}$

Contrariamente a la creencia de que la reducción en la masa del tumor después de la quimioradioterapia aumentará la probabilidad de resección anterior, un estudio polaco y dos metaanálisis mostraron que la reducción del tumor no tuvo impacto en la tasa de resección anterior. Sólo el estudio alemán de Sauer, Becker, Hohenberger, et al mostró un aumento significativo en la preservación del esfínter. (11)

En el presente estudio se observa un downstaging positivo en pacientes que recibieron quimioterapia de inducción como en los que no la recibieron, el porcentaje es mayor en los pacientes a los que se les administró quimioterapia de inducción sin embargo la diferencia no es estadísticamente significativa. Además se observa que en la mayoría de los pacientes el downstaging no pudo determinarse. 
Luego de que los pacientes reciben el ciclo de quimioradioterapia, ya sea sin previa quimioterapia de inducción o con previa quimioterapia de inducción, se realiza un estadiaje patológico que determina la respuesta del paciente previo a una resección. La información recolectada de los pacientes mostró que ninguno de ellos llegó a tener una respuesta patológica completa (yPT0, yPN0), más bien como se observa en la Tabla II $30 \%$ de los pacientes tuvieron un yPT indefinido y $50 \%$ de los pacientes un yPN indefinido. Todos los pacientes fueron sometidos a una resección, que como bien es sabido genera ciertos riesgos y condiciones no deseadas en los pacientes tales como la pérdida de materia fecal espontánea, por lo que se ha implementado la posibilidad de únicamente tratar a los pacientes con quimioterapia y luego esperar antes de ser sometidos a una cirugía radical. ${ }^{(25)}$

El metaanálisis elaborado por Li et al., que estudia la sobrevida libre de progresión en pacientes que mostraron una respuesta patológica completa luego de ser tratados únicamente con quimioterapia neoadyuvante en comparación con pacientes que fueron sometidos a cirugías de resección, llegó a la conclusión que no hay diferencia en la supervivencia a largo plazo, la aparición de metástasis distal, la sobrevida libre de progresión y la sobrevida global en pacientes tratados únicamente con quimioterapia neoadyuvante en comparación con aquellos que se sometieron a cirugía radical luego de la quimioterapia. Sin embargo, los pacientes tratados únicamente con quimioterapia neoadyuvante están asociados a presentar un mayor riesgo de recurrencia local. ${ }^{(12)}$ Por lo que se ve una relación directa en la sobrevida libre de progresión y una respuesta patológica completa.

En el caso de este estudio, la probabilidad de supervivencia libre de progresión demostró ser muy favorable.

Aunque teóricamente se espera que aproximadamente el $40 \%$ de los pacientes que se presentan con enfermedad en estadio II o III tengan una recurrencia de la enfermedad después de la terapia primaria, que más del $90 \%$ de las recidivas se desarrollen dentro de los primeros tres a cinco años luego del tratamiento y que la mayoría de los pacientes que desarrollen la enfermedad recurrente mueran de esta causa; ${ }^{(26)}$ los resultados del estudio presentan una diferencia notable ya que solo un $20 \%$ de los pacientes presentó recurrencia. De la misma manera, la probabilidad de su- pervivencia libre de progresión mostró ser alta ya que aproximadamente a los 2 meses se ve una recaída en únicamente 2 pacientes, a los 25 meses la recaída de un solo paciente al igual que a los 40 meses.

Cuadro 1. Características generales de la población en estudio

\begin{tabular}{|c|c|}
\hline Variable & $\begin{array}{l}\text { Número } \\
(\%)\end{array}$ \\
\hline Edad media (años) & $\begin{array}{ll}56,8 & \pm \\
11,12 & \\
\end{array}$ \\
\hline \multicolumn{2}{|l|}{ Sexo } \\
\hline Masculino & $11(55)$ \\
\hline Femenino & $9(45)$ \\
\hline \multicolumn{2}{|l|}{$\mathbf{T}$} \\
\hline $\mathrm{X}$ & $2(10)$ \\
\hline 1 & $1(5)$ \\
\hline 2 & $1(5)$ \\
\hline 3 & $13(65)$ \\
\hline 4 & $3(15)$ \\
\hline \multicolumn{2}{|l|}{$\mathbf{N}$} \\
\hline $\mathrm{X}$ & $13(65)$ \\
\hline 1 & $4(20)$ \\
\hline 2 & $3(15)$ \\
\hline \multicolumn{2}{|l|}{ M } \\
\hline 0 & $20(100)$ \\
\hline \multicolumn{2}{|l|}{ ECOG } \\
\hline 0 & $13(65)$ \\
\hline 1 & $6(30)$ \\
\hline 2 & $1(5)$ \\
\hline Inducción & $6(30)$ \\
\hline \multicolumn{2}{|l|}{ Tratamiento de Inducción } \\
\hline No recibieron & $14(70)$ \\
\hline 5-FU (5-flurouracilo) & $2(10)$ \\
\hline FOLFOX (5-flurouracilo+oxaliplatino) & $1(5)$ \\
\hline XELOX (capecitabina+oxaliplatino) & $3(15)$ \\
\hline \multicolumn{2}{|l|}{ Número de ciclos } \\
\hline No recibieron & $15(75)$ \\
\hline 3 & $2(10)$ \\
\hline 4 & $2(10)$ \\
\hline 6 & $1(5$ \\
\hline
\end{tabular}

Fuente: HSJD

Cuadro 2. Resultados de la respuesta al tratamiento de la población en estudio

\begin{tabular}{|lc|}
\hline Variable & Número (\%) \\
\hline $\begin{array}{l}\text { Tipo de resección } \\
\text { RAB (Resección abdomi- }\end{array}$ & $12(60)$ \\
nal baja) & $8(40)$ \\
RAP (Resección ad- & 8 \\
\hline
\end{tabular}




\begin{tabular}{|lc|}
\hline bominoperianal) & \\
IPT & \\
Indefinido & $6(30)$ \\
1 & $2(10)$ \\
2 & $2(10)$ \\
3 & $10(50)$ \\
\hline yPN & \\
Indefinido & $10(50)$ \\
0 & $5(25)$ \\
1 & $3(15)$ \\
2 & $2(10)$ \\
\hline Resección & \\
Indefina & $1(5)$ \\
Completa & $14(70)$ \\
Incompleta & $5(25)$ \\
\hline Recurrencia & \\
Si & $4(20)$ \\
No & $16(80)$ \\
\hline Pacientes fallecidos & \\
No & $19(95)$ \\
Si & $1(5)$ \\
\hline
\end{tabular}

Fuente: HSJD,

Cuadro 3. Porcentaje de pacientes con "downstaging"

\begin{tabular}{|lc|}
\hline Variable & $\begin{array}{c}\text { Número } \\
(\%)\end{array}$ \\
\hline $\begin{array}{l}\text { Downstaging descono- } \\
\text { cido }\end{array}$ & \\
Pacientes sin inducción & $3(21,4)$ \\
Pacientes con inducción & $3(50)$ \\
Total & $6(30)$ \\
\hline Downstaging positivo & \\
Pacientes sin inducción & $4(28,6)$ \\
Pacientes con inducción & $2(33,3)$ \\
Total & $6(30)$ \\
\hline Downstaging negativo & \\
Pacientes sin inducción & $7(50)$ \\
Pacientes con inducción & $1(16,7)$ \\
Total & $8(40)$ \\
\hline
\end{tabular}

Fuente: HSJD

\section{CONCLUSIONES}

Los resultados analizados en este estudio como la reducción de la masa tumoral, el dowstaging y la preservación del esfínter demostraron ser factores positivos en la sobrevida libre de progresión y la baja probabilidad de recurrencia de los pacientes. Esto indica que las técnicas empleadas para tratar el cáncer colonorrectal en Hospital San Juan de Dios han estado bien dirigidas a lo largo de estos 5 años, ya que los resultados generales mostraron tener beneficios en la población.

Sin embargo, por la cantidad de pacientes incluidos en el estudio, no se puede afirmar con este estudio que la quimioterapia de inducción, seguida de quimioradioterapia y cirugía genere mayores beneficios sobre la quimioradioterapia seguida de cirugía. Incluso no se puede demostrar que exista una similitud entre ambos.

En cuanto a la toxicidad de la quimioterapia de inducción, ninguno de los pacientes que la recibió presentó ningún efecto adverso que fuera documentado. Sin embargo, la población es tan poco representativa que no se puede concluir que la quimioterapia de inducción cause o no mayor toxicidad a los pacientes que la reciben.

Se recomienda ampliar la población en estudio, ya que aunque se deseaba ver la sobrevida de los pacientes luego de cinco años esta población llegó a ser tan pequeña que los resultados no pudieron ser comparados ni mostraron ser estadísticamente significativos.

Es importante que los especialistas estudien y analicen los nuevos enfoques donde se da seguimiento al paciente luego de la quimioterapia neoadyuvante y así ver si incluso puede haber una sobrevida libre de progresión sin que el paciente sea expuesto a una cirugía radical. Además, se debe analizar la posibilidad de incluir la quimioterapia de inducción como un estándar en aquellos pacientes con masas tumorales que pueden ser reducidas con más facilidad.

\section{BIBLIOGRAFÍA}

1. Davis LE, Sun W, Medina PJ. Colorectal Cancer. En Pharmacotherapy: A Pathophysiologic Approach. DiPiro JT, Talbert RL, Yee GC, Matzke GR, Wells 
BG, Posey L. (Eds). McGraw-Hill Companies Inc, New York, United States. 9ed, 2014.

2. Holle LM, Clement JM, Davis LE. Colorectal Cancer. En Pharmacotherapy: A Pathophysiologic Approach. DiPiro JT, Talbert RL, Yee GC, Matzke GR, Wells BG, Posey L. (Eds). McGraw-Hill Companies Inc, New York, United States. 10ed, 2017.

3. Hanahan D, Weinberg R. Hallmarks of cancer: The next generation. Cell. 2014; 144: 646-674.

4. NCCN: Clinical Practice Guidelines in Oncology. Rectal Cancer. 2016; 41187.

5. Samalavicius $\mathrm{N}$, Ambrazevicius M, Kilius A, Petrulis K. Transanal endoscopic microsurgery for early rectal cancer: single center experience. Wideochir Inne Tech Maloinwazyjne. 2014; 9 (4): 603-607

6. Ghosn M, Kourie HR, Abdayem P, Antoun J, Nasr D. Anal cancer treatment: Current status and future perspectives. World J Gastroenterol. 2015; 21(8): 2294-2302

7. Hospital Universitario Reina Sofia. Subcomisión clínica de tumores digestivos: Protocolo cáncer colorectal. España: Universidad de Andalucía. 2013

8. Glynne-Jones R, Grainger J, Harrison M, Ostler P, Makris A. Neoadjuvant chemotherapy prior to preoperative chemoradiation or radiation in rectal cancer: should we be more cautious? British Journal of Cancer. 2006; 94: 363 $-371$

9. Glynne-Jones R, Ian Chau I. Neoadjuvant therapy before surgical treatment. EJC Supplements. 2013; 11: 45-59

10. Tepper JE, O'Connell M, Niedzwiecki $\mathrm{D}$, et al. Adjuvant therapy in rectal cancer: analysis of stage, sex, and local control--final report of intergroup 0114. J Clin Oncol 2002; 20:1744-1750.

11. Sauer R, Becker H, Hohenberger W, et al. Preoperative versus postoperative chemoradiotherapy for rectal cancer. NEJM. 2004;351:1731-1740.

12. Li J, Li L, Yang L et al. Wait-and-see treatment strategies for rectal cancer patients with clinical complete response after neoadjuvant chemoradiotherapy: a systematic review and meta-analysis. Oncotarget. 2016; 7(28): 44857-44870

13. Rahbari NN, Elbers H, Askoxylakis V, et al. Neoadjuvant radiotherapy for rectal cancer: meta-analysis of randomized controlled trials. Ann Surg Oncol 2013; 20:4169-4182.

14. Gunderson LL, Sargent DJ, Tepper JE, et al. Impact of $\mathrm{T}$ and $\mathrm{N}$ stage and treatment on survival and relapse in adjuvant rectal cancer: a pooled analysis. J Clin Oncol 2004; 22:1785-1796.

15. GLOBOCAN 2012: Estimated cancer incidence, mortality and prevalence worldwide 2012. Disponible en: http://globocan.iarc.fr/old/burden.asp? selection pop $=44188 \&$ Text$\mathrm{p}=$ Costa + Rica\&selection cancer $=5060$ \&Text$\mathrm{c}=$ Colorectum\&pYear $=3 \&$ type $=0 \&$ window $=1 \&$ submit $=\% \mathrm{C} 2 \% \mathrm{~A} 0$ Execute

16. Registro Nacional de Tumores. Ministerio de Salud (2016). Situación epidemiológica del cáncer actualizado: Estadística de cáncer-Costa Rica. Disponible en: https://www.ministeriodesalud.go.cr/ind ex.php/vigilancia-de-lasalud/estadisticas-y-bases-dedatos/estadisticas/estadistica-de-cancerregistro-nacional-tumores/2722situacion-epidemiologica-del-cancer/file

17. Newcamb PA, carbine PP. Cancer treatment and age: patient perspectives. J Natl Cancer Inst. 1993; 85: 1580

18. Extermann M, Boler I, reich RR et al. Predicting the risk of chemotherapy tox- 
icity in older patients: the Chemotherapy Risk Assessment scale for High Age Patients (CRASH) score. Cancer. 2012; 118: 3377

19. Hurria A, Tagawa K, Mohile SG et al. Predicting chemotherapy toxicity in older adults with cancer-. A prospective multicenter study. J Clin Oncol 2011; 29: 3457

20. Fernández-Martos C, Pericay C, Aparicio J et al. Phase II, randomized study of concomitant chemoradiotherapy followed by surgery and adjuvant capecitabine plus oxaliplatin (CAPOX) compared with induction CAPOX followed by concomitant chemoradiotherapy and surgery in magnetic resonance imaging-defined, locally advanced rectal cancer. J Clin Oncol. 2010;28(5):859-65.

21. Mayer RJ. Lower Gastrointestinal Cancers. En Harrison's Principles of Internal Medicine. Kasper D, Fauci A, Hauser S, Longo D, Jameson J, Loscalzo J (Eds). McGraw-Hill Companies Inc, New York, United States. 19e, 2016.

22. Bhatti A, Waheed A, Hafeez A et al. Can induction chemotherapy before concurrent chemoradiation impact circumferential resection margin positivity and survival in low rectal cancers? Asian Pac J Cancer Prev. 2015;16(7):2993-8.

23. Chau I, Brown G, Cunningham D, et al. Neoadjuvant Capecitabine and Oxaliplatin Followed by Synchronous Chemoradiation and Total Mesorectal Excision in Magnetic Resonance Imaging-Defined Poor-Risk Rectal Cancer. J Clin Oncol. 2006,24:668-674.

24. Nogué M, Salud A, Vicente P, Arriví A Roca JM Losa F Ponce J et al. Addition of Bevacizumab to XELOX Induction Therapy Plus Concomitant Capecitabine-Based Chemoradiotherapy in Magnetic Resonance ImagingDefined Poor-Prognosis Locally Ad- vanced Rectal Cancer: The AVACROSS Study. Oncologist. 2011;16(5):614-20.

25. Xynos E, Tekkis P, Gouvas $\mathrm{N}$ et al. Clinical practice guidelines for the surgical treatment of rectal cancer: a consensus statement of the Hellenic Society of Medical Oncologists (HeSMO). Ann Gastroenterol. 2016; 29(2): 103-126.

26. Haggstrom D, Cheung W. Approach to the long-term survivor of colorectal cancer.Wolters Kluer, UptoDate. 2015.

\section{CONFLICTO DE INTERÉS Y/O AGRADECIMIENTOS}

Los autores declaran que no existió ningún conflicto de interés en el presente reporte. 